\title{
Flúter auricular en paciente con isomerismo izquierdo y vena cava inferior izquierda interrumpida
}

Atrial flutter in a patient with left isomerism and left-sided inferior vena cava interruption

\author{
Richard Soto ${ }^{1}$, MD, Luis C. Sáenz², Fermín García ${ }^{3}$, MD, \\ Diego Rodriguez ${ }^{2}, M D$ \\ 'Médico Cardiólogo, residente de electrofisiología. Centro Internacional de Arritmias "Andrea Natale", Fundación Cardioinfantil, \\ Bogotá,Colombia. \\ ${ }^{2}$ Médico electrofisiólogo, Centro Internacional de Arritmias "Andrea Natale”, Fundación Cardioinfantil, Bogotá, Colombia. \\ ${ }^{3}$ Médico electrofisiólogo, División de Cardiología, Departamento de Medicina, Hospital de la Universidad de Pennsylvania, Filadelfia, EE UU.
}

\begin{abstract}
Resumen
Se comunica la ablación de un flúter auricular con una longitud de ciclo de $260 \mathrm{~ms}$ en un paciente de 28 años con isomerismo izquierdo y vena cava inferior izquierda interrumpida. Mediante encarrilamiento, demostramos la participación del istmo suprahepáticotricuspídeo en el circuito de macroreentrada. Realizamos líneas de ablación con radiofrecuencia que detuvieron la arritmia primaria y culminó en ritmo de escape nodal.
\end{abstract}

Palabras clave. Flúter Auricular; Isomerismo Izquierdo; Dextrocardia,

Abstract

We report the ablation of an atrial flutter with a $260 \mathrm{~ms}$ cycle length in a 28-year-old patient with left isomerism and interrupted left inferior vena cava. We showed the participation of the suprahepatic - tricuspid isthmus in the macro reentry circuit by the entrainment maneuver.

Keywords. Atrial Flutter; Left Isomerism; Dextrocardia.

An Fac med. 2016;77(2):163-6 / http://dx.doi.org/10.15381/anales.v77i2.11821

\section{INTRODUCCIÓN}

El tratamiento invasivo de las taquiarritmias en pacientes adultos con cardiopatía congénita plantea un reto al electrofisiólogo, debido a la complejidad anatómica, accesos vasculares y hallazgos electrofisiológicos inesperados ${ }^{(1)}$.

Los pacientes con isomerismo atrial izquierdo representan el $23 \%$ de la taquiarritmias supraventriculares, siendo más frecuentes la fibrilación auricular y las macroreentradas ${ }^{(2,3)}$. Sin embargo, más del $54 \%$ presentará bradiarritmias como disfunción sinusal asociada con agenesia o hipoplasia de nodos sinusales ectópicos ${ }^{(4)}$.
Se reporta el caso de un paciente con isomerismo izquierdo, vena cava inferior izquierda interrumpida y flúter auricular (FlA) dependiente del istmo suprahepático-tricuspídeo, quien presentó ritmo de escape nodal persistente durante las 48 horas postablación inmediata, por lo cual requirió implante de marcapaso bicameral definitivo.

\section{REPORTE DE CASO}

Paciente masculino de 28 años, con situs inversus, dextrocardia, isomerismo izquierdo. Fue intervenido quirúrgicamente para cierre de comunicación interauricular (CIA) a los 5 años de edad.
A los 22 años se le realizó cambio de válvula mitral por prótesis mecánica y cirugía de maze. Presentaba una historia de más de un año con palpitaciones taquicárdicas asociadas a deterioro de clase funcional, siendo refractario a la amiodarona.

El electrocardiograma (gráfica 1) mostró ritmo de flúter auricular. El ecocardiograma documentó situs inversus, fracción de eyección del ventrículo izquierdo de $38 \%$, sin shunt residual. La tomografía axial computarizada tuvo hallazgos compatibles con situs inversus e isomerismo izquierdo (ausencia de segmento hepático de la vena cava inferior izquierda, ambos pulmones bilobulados y poliesplenia), conexión de 


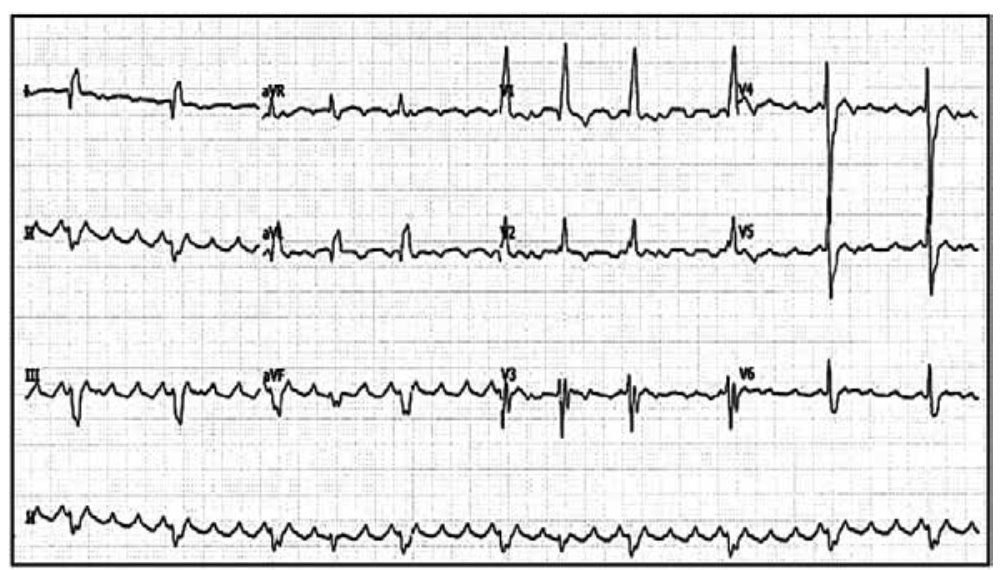

Gráfica 1. Electrocardiograma basal en ritmo de flúter auricular con conducción auriculoventricular variable, frecuencia cardiaca promedio $721 \mathrm{pm}$, ondas de activación atrial regulares positivas en derivaciones DII, DIII y AVF, negativas en $\mathrm{V} 1$, con transición a la positividad desde $\mathrm{V} 4 \mathrm{a}$ V6. venas suprahepáticas y vena cava superior a la aurícula derecha $(\mathrm{AD})$.

Se realizó ablación del FlA empleando sistema de mapeo tridimensional (NavX; St Jude Medical, Inc.). Se practicó venografía desde las venas ilíacas para observar la vena cava inferior izquierda, interrumpida a nivel de la vena renal derecha y continua con el sistema venoso hemiácigos, para llegar a la vena cava superior que drena en la AD (gráfica 2). Se realizó reconstrucción tridimensional de $\mathrm{AD}$ con un catéter circular (Inquiry AFocus II, St Jude Medical, Inc), así como mapa de voltaje con áreas de cicatriz en segmento inferior de pared lateral de $\mathrm{AD}$, el cual corresponde a atriotomía. Además, se observó área de cicatriz en el tercio inferior del séptum interauricular, que corresponde al parche del cierre de CIA (gráfica 3A).

Se encontró ritmo de FlA con longitud de ciclo (LC) de 260 ms. Utilizamos un catéter de ablación irrigado (Therapy Cool Flex, St Jude Medical, Inc), para posicionarlo en el istmo suprahepático-tricuspídeo lateral y en el cual se realizó encarrilamiento con una LC de $240 \mathrm{~ms}$, observando captura auricular, fusión oculta e intervalo postestimulación (IPE) de 263 ms (gráfica 3B). Luego se practicó encarrilamiento desde el

practicar línea de ablación del istmo suprahepático-tricúspideo lateral, eliminando así la arritmia (gráfica 3D) y pasando a asistolia, por lo que requirió estimulación atrial de forma transitoria, pasando a ritmo de la unión. Se realizó maniobras confirmando bloqueo bidireccional del istmo suprahepático-tricúspideo y sobreestimulación atrial, sin inducir taquiarritmias atriales.

Durante las 48 horas de observación postablación persistió en ritmo nodal, con 2 episodios de fibrilación auricular paroxística sintomática con respuesta ventricular alta, requiriendo reinicio de amiodarona. Dada la persistencia del ritmo nodal y la necesidad de mantener antiarrítmicos, se implantó un marcapaso bicameral.

istmo suprahepático tricúspideo septal con IPE de 283 ms (gráfica 3C).

Realizamos línea de ablación con radiofrecuencia en el istmo suprahepático-tricúspideo en su porción media. No siendo efectiva, se procedió a
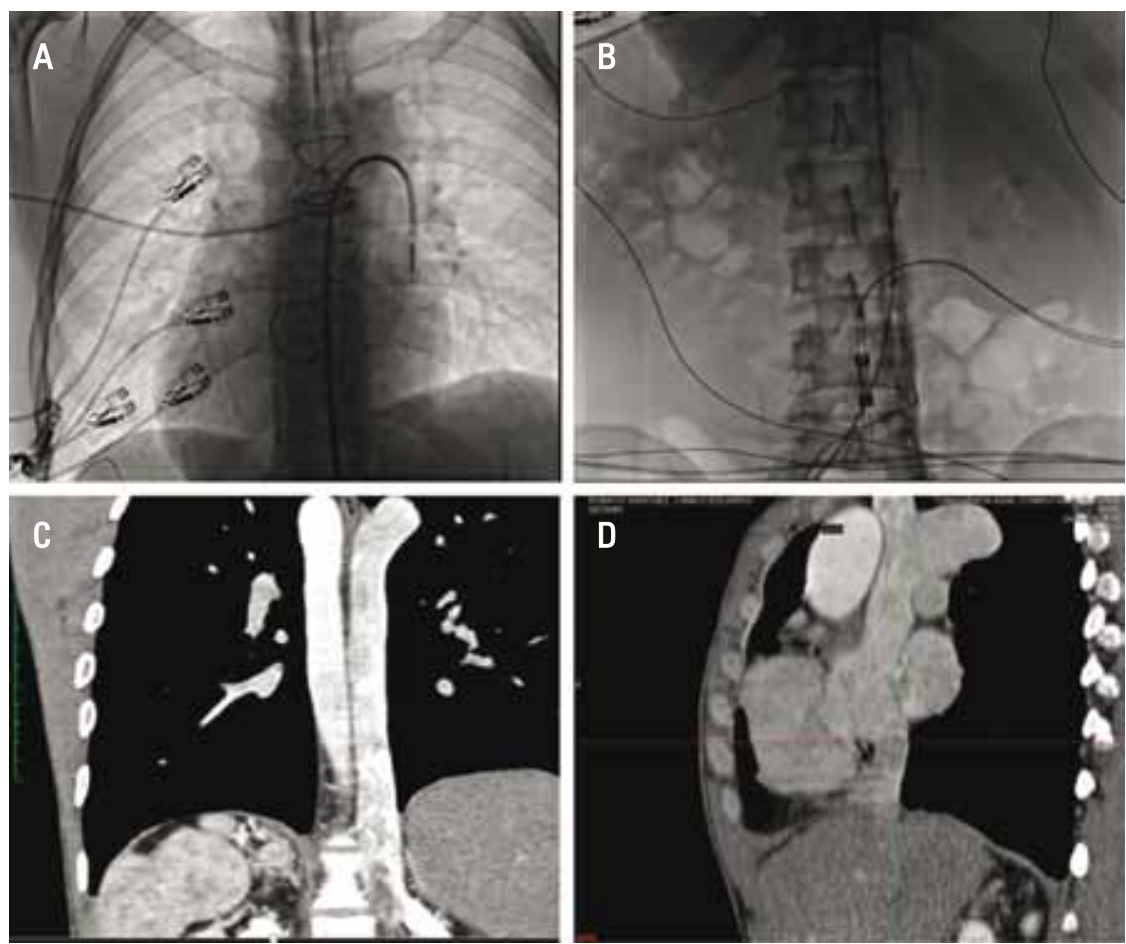

Gráfica 2. Anatomía cardiaca. A. Dextrocardia. B. VCl izquierda interrumpida. C. VCl izquierda interrumpida continuada con sistema hemiacigos. D. Drenaje directo de venas suprahepáticas en AD. 

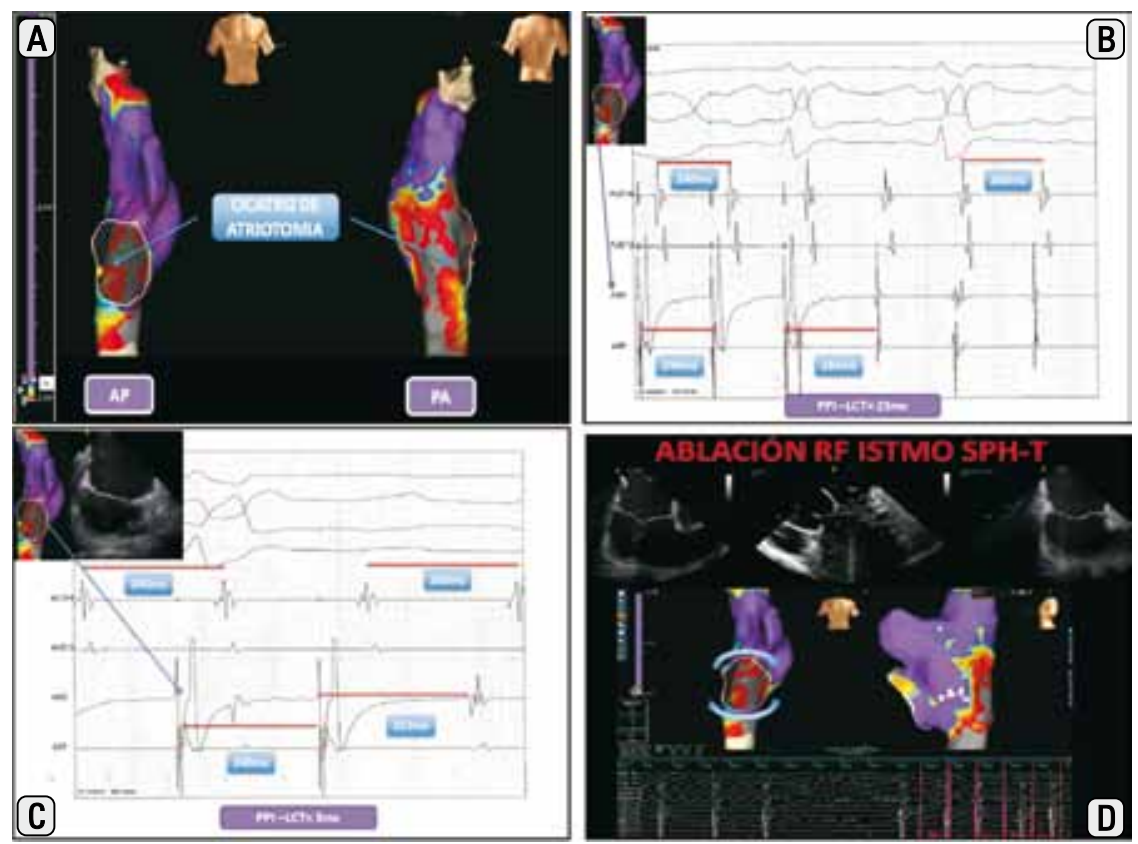

Gráfica 3. A. Mapa de voltaje de AD; observamos área de cicatriz correspondiente a atriotomia.

B. Encarrilamiento del FIA estimulando desde el istmo suprahepático tricuspídeo lateral. C. Encarrilamiento del FIA estimulando desde el istmo suprahepático tricuspídeo septal. D. Línea de ablación efectiva en istmo suprahepático tricuspídeo lateral; obsérvese que al eliminar el flúter auricular, el paciente pasó a asistolia y requirió estimulación atrial.

cardiopatía congénita, con heterogeneidad de presentación clínica dependiente de las malformaciones cardiacas congénitas asociadas. La presencia de interrupción de la porción suprarrenal de la vena cava inferior asociada a poliesplenia es sugerente de isomerismo atrial izquierdo ${ }^{(1)}$.

La sobrevida se ha incrementado debido a las mejores técnicas quirúrgicas y manejo médico, observándose un mayor número de adultos con estas malformaciones congénitas. Existe mayor riesgo del desarrollo de arritmias debido al sustrato anatómico de base y cicatrices resultado de cirugías previas ${ }^{(2)}$. La incidencia de taquicardias supraventriculares llega hasta $23 \%$, siendo más frecuente la fibrilación auricular seguida de las taquiarritmias por macroreentrada, las cuales requieren barreras anatómicas o cicatrices generadas por cirugía cardiaca previa ${ }^{(3)}$.

La interrupción de la vena cava inferior seguida con el sistema ácigos o hemiácigos es observada en pacientes con isomerismo izquierdo debido a una falta en la formación de la anastomosis subcardinal-hepática y subsecuente atrofia de la vena subcardinal, generando que la vena cava inferior infrarrenal se continúa con el sistema venoso ácigos si la vena cava inferior está a la derecha, o sistema venoso hemiácigos si la vena cava inferior está a la izquierda ${ }^{(5)}$.

La complejidad de la anatomía en pacientes con cardiopatía congénita plantea un reto al electrofisiólogo. Este caso ilustra el rol de las pruebas de imágenes, tales como la tomografía computarizada, fluoroscopia, ecografía intracardiaca, sistema de mapeo electroanatómico tridimensional, como ayuda al electrofisiólogo para entender las estructuras anatómicas complejas, teniendo un procedimiento de ablación seguro y exitoso ${ }^{(1)}$.

Diferentes reportes muestran que, en el seguimiento a largo plazo, más de $54 \%$ de los pacientes con isomerismo izquierdo presentará bradiarritmias, como disfunción sinusal ${ }^{(4)}$, llegando a tener algún grado de bloqueo auriculoventricular ${ }^{(6)}$. Los estudios histológicos han demostrado nodo sinusal ausente, hipoplásico y ectópicos ubicados en el segmento alto, medio o bajo de la pared lateral de la $\mathrm{AD}^{(7)}$, asociado a ritmo atrial o escape nodal ${ }^{(8)}$.

Durante la ablación del FlA, el paciente pasó a asistolia transitoria y luego a ritmo nodal. En las 48 horas postablación persistió en ritmo nodal, asociando episodios de fibrilación auricular paroxística sintomática que requirió amiodarona. Por lo tanto, se decidió el implante de marcapaso bicameral.

Este caso enfatiza las características inusuales de las arritmias en pacientes con isomerismo izquierdo y vena cava inferior izquierda interrumpida, y las estrategias de abordaje dada la inusual anatomía, difícil acceso venoso y el desenmascaramiento de agenesia o disfunción del nodo sinusal.

\section{REFERENCIAS BIBLIOGRÁFICAS}

1. Haegeli LM, Greutmann M, Wolber T, Appenzeller P, Gaemperli O, Brunckhorst C, et al. Complex cardiac anatomy and catheter access: the role of imaging in patients referred for catheter ablation. Europace. 2011Aug;13(8):1203-5. doi:10.1093/ europace/eur059.

2. Suman-Horduna I, Babu-Narayan SV, Ueda A, Mantziari L, Gujic M, Marchese P, et al. Magnetic navigation in adults with atrial isomerism (heterotaxy syndrome) and supraventricular arrhythmias. Europace. 2013 Jun;15(6):877-85. doi:10.1093/ europace/eus384.

3. Miyazaki A, Sakaguchi $H$, Ohuchi $H$, Yamamoto T, Igarashi T, Negishi J, et al. The incidence and characteristics of supraventricular tachycardia in left atrial isomerism: a high incidence of atrial fibrillation in young patients. Int $\mathrm{J}$ Cardiol. 2013 Jun 20;166(2):375-80. doi:10.1016/j. ijcard.2011.10.118

4. Wu MH,Wang JK, Lin JL, Lai LP, Lue HC, Hsieh FJ. Cardiac rhythm disturbances in patients with left atrial isomerism. Pacing Clin Electrophysiol 2001;24:1631-8.

5. Morató Lopez AE, Quiroz Castro O, Ramirez Arias JL. Anomalias congénitas de la vena cava inferior: el rol de la tomografía computada multidetector. Artículo de Revisión. Anales de Radiología Mexico. 2012;2:104-13

6. Momma K, Takao A, Shibata T. Characteristics and natural history of abnormal atrial rhythms in left isomerism. Am J Cardiol. 1990;65:231-6.

7. Hassem Sobrinho S, Camacho Moscardini A, Croti, Alexandre Croti U, Henrique de Marchi, C, Fernandes de Godoy M. Disfunção sinusal em paciente com isomerismo atrial esquerdo Arquivos Brasileiros de Cardiologia 2006; 87(4): 122-123. 
An Fac med. 2016;77(2):163-6

8. Dickinson DF, Wilkinson JL, Anderson KR, Smith A, Ho SY, Anderson RH. The cardiac conduction system in situs ambiguous. Circulation. 1979;59:879-85.

Artículo recibido el 26 de noviembre de 2015 y aceptado para publicación el 17 de diciembre de 2015.

Conflicto de interés:

No existe en la presente comunicación.

Correspondencia:

Richard Soto.

Centro Internacional de Arritmias.

Calle 163A No 13B-60, Bogotá Colombia.

Teléfono: (571)6672727 extensión 41401

Correo electrónico: cardsot@gmail.com 